\title{
Massa paranasal: Dacrioestenose congênita? - Relato de caso
}

\author{
Paranasal mass: Congenitaldacryostenosis?-Case report
}

\author{
Claudia Akemi Shiratori ${ }^{1}$ \\ Silvana Artioli Schellini ${ }^{2}$ \\ Aristides Palhares ${ }^{3}$ \\ Ricardo de Campos Schellini ${ }^{4}$ \\ Seizo Yamashita ${ }^{4}$
}

\section{RESUMO}

O objetivo deste é descrever uma criança portadora de massa paranasal, atentando para a importância dos diagnósticos diferenciais. Relato do caso: ACS, 6 meses, sexo feminino, desde o nascimento apresentando abaulamento não inflamatório, no canto medial do olho esquerdo, lacrimejamento e hiperemia no olho direito. Ao exame apresentava fenômeno de Bell negativo bilateral, lagoftalmo à direita, ulceração e opacidade corneana à direita; presença de lesão arredondada, de superfície lisa no canto medial do olho esquerdo, sem sinais inflamatórios, medindo aproximadamente 2 cm de diâmetro, não pulsátil. À palpação, a lesão era elevada, de consistência fibroelástica, imóvel, indolor, irredutível. À propedêutica das vias lacrimais, não havia refluxo à compressão, o teste de Milder foi negativo em ambos olhos e as vias apresentavam-se pérvias à dacriocistografia. $\mathrm{O}$ exame tomográfico revelou tratar-se de meningocele fronto-etmoidal. Comentários: Os autores chamam a atenção para a adequada semiologia para a investigação das massas paranasais, a fim de se instituir o adequado tratamento.

Descritores: Obstrução dos ductos lacrimais/congênito; Encefalocele/diagnóstico; Meningocele/diagnóstico; Seio etmóide/patologia; Seios paranasais/patologia; Diagnóstico diferencial; Criança; Relato de caso

\section{INTRODUÇÃO}

A obstrução nasolacrimal congênita (ONLC) ocorre em 6 a $20 \%$ dos neonatos ${ }^{(1-2)}$, ou até em 30 até $73 \%$ dos recém-nascidos a termo ${ }^{(3-4)}$.

A ONLC pode ser confundida com outras massas paranasais de ocorrência menos freqüientes, como dacriocistocele (amniocele e mucocele), hemangioma, meningoencefalocele nasal e cisto dermóide ${ }^{(3-4)}$.

As dacriocistoceles podem ser consideradas um espectro da ONLC, podendo ocorrer em ambos os $\operatorname{sexos}^{(3)}$. Trata-se de uma combinação entre obstrução da via lacrimal e obstrução retrógrada do fluxo pelo canalículo no nível da válvula de Rosenmüller, ou seja, uma obstrução distal e proximal da via lacrimal, com subseqüente dilatação do saco lacrimal, de coloração azulada, podendo ser confundida com uma lesão vascular ${ }^{(4)}$.

A dacriocistocele pode ser chamada mucocele do saco lacrimal, quando preenchida por muco, ou amniocele (ou amniotocele), quando preenchida por fluido amniótico ${ }^{(4)}$.

Outro termo empregado é cisto lacrimal congênito, considerado por alguns um termo preferível para descrever a anormalidade, quando não é possível estabelecer a patogênese ${ }^{(3)}$.

A dacriocistocele é uma lesão cística tensa, de coloração azul-acinzentada, localizada logo abaixo do tendão cantal medial, podendo apresentar características inflamatórias, até celulite ${ }^{(4)}$. O tratamento recomendado, quando não há cura espontânea ou após sondagem, é a marsupialização do cisto ${ }^{(3)}$. 
Outros diagnósticos possíveis seriam as meningoencefaloceles anteriores ou posteriores que são lesões raramente observadas. A incidência das encefaloceles anteriores é mais elevada em asiáticos (Ocidente: 1: 35000 nascidos vivos/ano e na Tailândia 1: 5000 nascidos vivos/ano ${ }^{(5)}$ ). Podem ser classificadas em: frontoetmoidal (nasofrontal, nasoetmoidal e nasoorbitária), nasolaríngea e orbital ${ }^{(6)}$.

O objetivo deste foi relatar o caso de uma criança diagnosticada inicialmente como portadora de ONLC, chamando a atenção para os diagnósticos diferenciais.

\section{RELATO DO CASO}

ACSM, com idade de 6 meses, sexo feminino, foi encaminhada ao Ambulatório de Vias Lacrimais da Faculdade de Medicina de Botucatu com diagnóstico de dacrioestenose congênita à esquerda. A criança apresentava abaulamento no canto medial, ocupando a região paranasal à esquerda desde o nascimento, sem sinais inflamatórios e lacrimejamento em ambos os olhos, com hiperemia no olho direito.

Antecedentes Pessoais: atraso no desenvolvimento neuropsico-motor e síndrome convulsiva controlada.

Ao exame externo apresentava lesão arredondada, de superfície lisa, na região do canto medial esquerdo, sem sinais inflamatórios, medindo aproximadamente $2 \mathrm{~cm}$ de diâmetro (Figura 1). À palpação, a lesão era elevada, de consistência macia, imóvel, indolor, irredutível e não pulsátil.

$\mathrm{O}$ fenômeno de Bell era negativo em ambos os lados, lagoftalmo paralítico à direita, ulceração e opacidade corneana à direita e estrabismo convergente. Os pontos lacrimais apresentavam-se tópicos e pérveos, sem secreção. Não havia refluxo de secreção à expressão e o teste de Milder foi negativo bilateralmente.

A exclusão definitiva da obstrução lacrimal congênita decorreu da constatação de que as vias apresentavam-se pér-

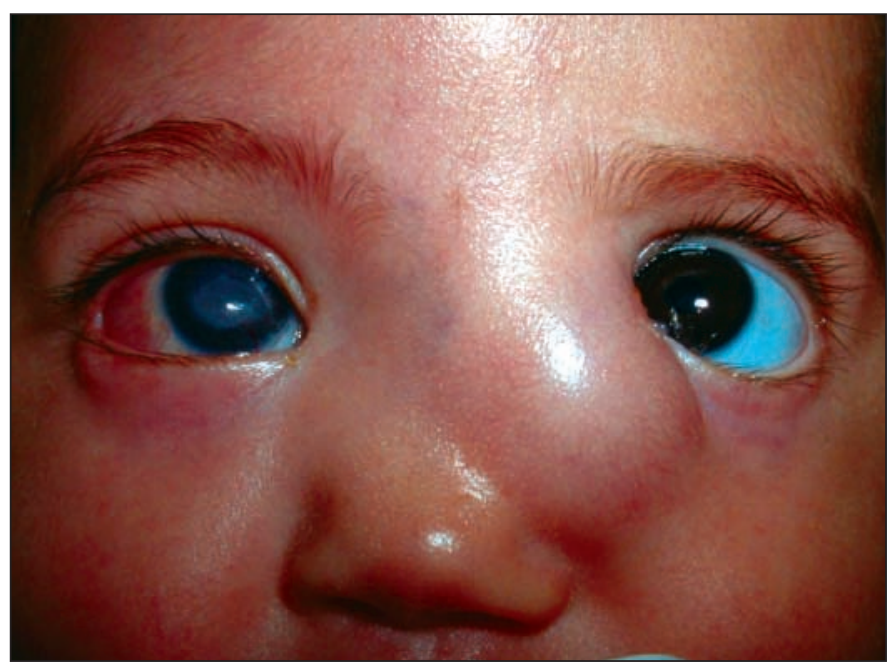

Figura 1 - Observar lesão cística abaulada no canto interno do olho esquerdo veas no exame dacriocistográfico, feito com contraste oleoso (Lipiodol).

A tomografia computadorizada de crânio e órbitas mostrou uma imagem cística de aproximadamente 2,0 X $2,0 \mathrm{~cm}$, com halo hiperdenso e hipodensidade central. A lesão se apresentava adjacente a uma falha óssea, caracterizada por uma descontinuidade entre o osso frontal e o etmóide esquerdo (Figura 2), confirmando o diagnóstico de uma meningocele fronto-etmoidal. Não se observou córtex cerebral herniado pela falha óssea.

A criança foi submetida a vários recobrimentos conjuntivais por causa da ulceração corneana, tendo também sido
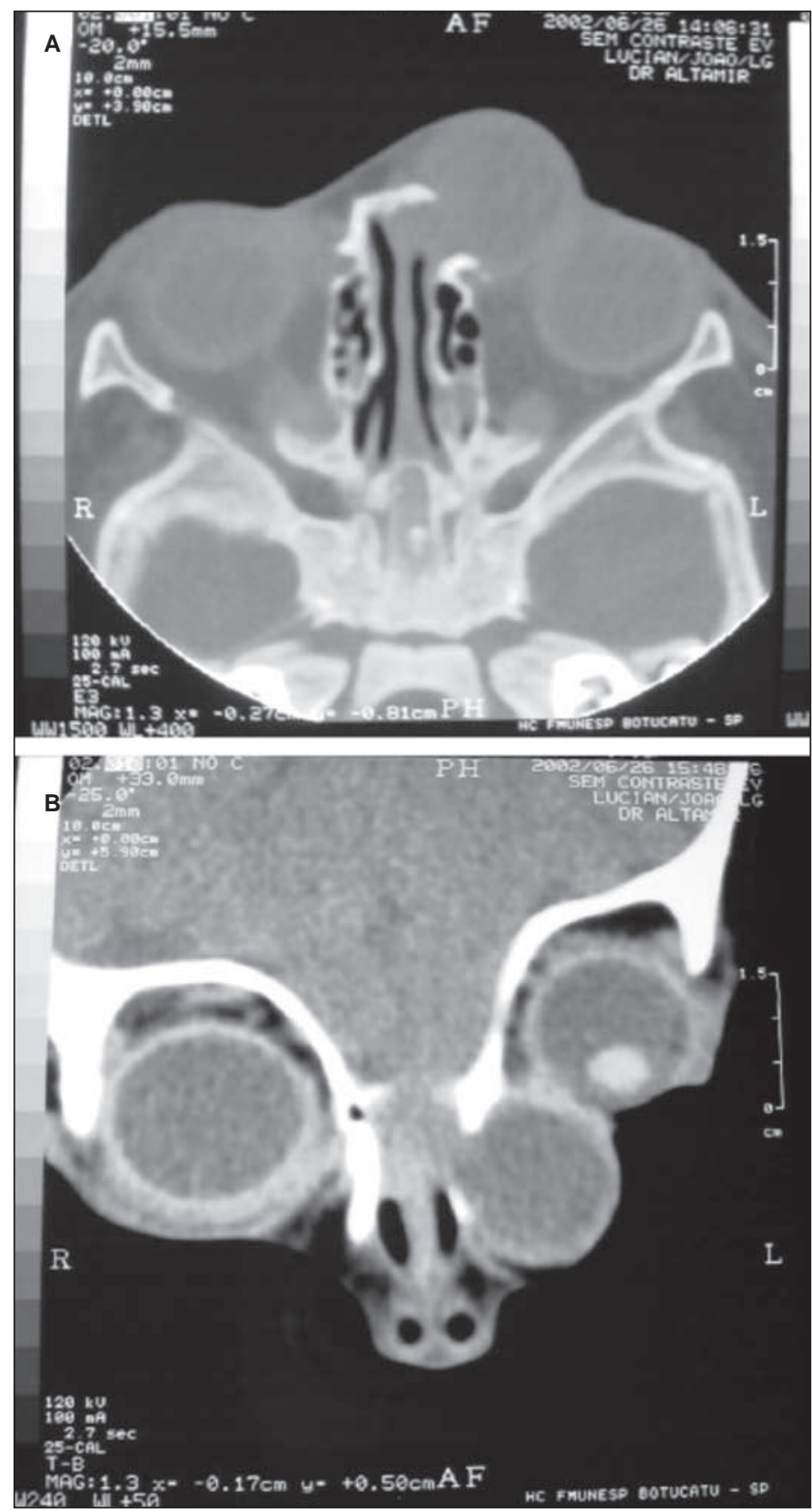

Figura 2 - Tomografia computadorizada de órbitas em corte axial (A) e coronal (B) demonstrando a lesão de aspecto cístico e descontinuidade óssea frontoetmoida 
operada para a correção da meningoencefalocele aos 15 meses de vida. Atualmente, mantém déficit neurológico, leucoma corneano à direita e o estrabismo. Está em tratamento fisioterápico e passa bem.

\section{COMENTÁRIOS}

A paciente deste relato apresentava massa paranasal e lacrimejamento, sem secreção ou sinais inflamatórios. Apresentava, ainda, lagoftalmo que poderia levar à exposição e úlcera corneana, gerando lacrimejamento ativo. Neste sentido, as alterações corneanas observadas comprovavam esta hipótese, diferenciando o lacrimejamento ativo da epífora.

Contudo, a própria lesão no canto medial poderia secundariamente causar dificuldade para a drenagem das lágrimas de modo indireto, por compressão da via lacrimal.

Chamava a atenção também o tamanho da massa e a ausência de sinais inflamatórios, inclusive sem nenhum quadro de dacriocistite aguda prévia.

Outros sinais de que a via lacrimal não estava envolvida no processo seriam a ausência de refluxo de secreção à expressão e o teste de excreção de corante (Teste de Milder) negativo.

Ainda assim, optou-se pela realização da dacriocistografia, procedimento que excluiu definitivamente a hipótese de ONLC.

A observação clínica das características da massa auxilia o diagnóstico. As meningoencefaloceles são mais comuns acima do tendão cantal medial, podendo ser pulsáteis, característica típica de lesões vasculares, como os hemangiomas. Neste caso, outros exames são necessários, como a ultra-sonografia que revela refletividade interna e o aspirado da lesão que é sanguinolento $^{(4)}$

Em se tratando de amniocele, o aspirado da massa é seroso, enquanto na mucocele, a secreção aspirada é mucosa, além de haver distensão aguda e inflamação mais freqüentes ${ }^{(4)}$.

No presente caso, o exame tomográfico foi decisivo para o diagnóstico, mostrando falha óssea que permitia a herniação da meninge para a região orbitária, caracterizando a lesão cística como meningocele. A tomografia computadorizada constata também o defeito ósseo e outras possíveis anomalias, como hidrocefalia, agenesia do corpo caloso, entre outras. A criança em questão apresentava atraso do desenvolvimento neuro-psico-motor, ainda em investigação.

O tratamento da meningoencefalocele é cirúrgico, por acesso externo, transcranial ou intranasal (nas encefaloceles basais); a morbidade pós-operatória ocorre por perda de líquido cefalo-raquidiano, infecção da ferida ou pulmonar ${ }^{(5)}$.

Caso não se suspeitasse de quadro diverso da ONLC, a sondagem poderia ter causado complicações como: lesão dos canalículos lacrimais, falso pertuito com infiltração de contraste nos tecidos adjacentes ${ }^{(7)}$, celulite pré-septal, sepsis pós-sondagem ${ }^{(1,8)}$ e bacteremia assintomática ${ }^{(8)}$.

Portanto, o diagnóstico das massas paranasais deve ser preciso, para que o adequado tratamento possa ser instituído.

\section{ABSTRACT}

Report of a child presenting a paranasal mass, and discussion of the importance of the differential diagnosis. Case report: ACS, 6 months old, female, presenting a non inflammatory nodulation on the left medial canthus; tearing and redness in the right eye since birth. On examination, there were bilateral lagophthalmos and corneal ulceration and opacity at the right side; on the left medial canthus there was a rounded lesion with a smooth surface, without inflammation, with an approximately 2-cm diameter. On palpation, the lesion was elevated, fibroelastic, non-mobile, painless, and irreductible. Tear or discharge reflux was absent on lacrimal pathway compression, Milder's test was negative on both sides. Dacryocystographic examination showed normal lacrimal drainage of the paranasal sinus system. Computadorized tomography revealed a fronto-ethmoidal meningocele. Comments: The authors emphasize the importance of the investigation of paranasal masses, in order to apply adequate therapy.

Keywords: Lacrimal duct obstruction/congenital; Encephalocele/diagnosis; Meningocele/diagnosis; Ethmoid sinus/ pathology; Paranasal sinus/pathology; Differential diagnosis; Child; Case report

\section{REFERÊNCIAS}

1. Zwaan J. Treatment of congenital nasolacrimal duct obstruction before and after the age of 1 year. Ophthalmic Surg Lasers. 1997;28(11):932-6.

2. Young JD, MacEwen CJ. Managing congenital lacrimal obstruction in general practice. BMJ. 1997;315(7103):293-6. Review.

3. Berkowitz RG, Grundfast KM, Fitz C. Nasal obstruction of the newborn revisited: clinical and subclinical manifestations of congenital nasolacrimal duct obstruction presenting as a nasal mass. Otolaryngol Head Neck Surg. 1990;103(3):468-71.

4. Ogawa GS, Gonnering RS. Congenital nasolacrimal duct obstruction. J Pediatr. 1991;119(1 Pt 1):12-7.

5. Mahapatra AK, Suri A. Anterior encephaloceles: a study of 92 cases. Pediatr Neurosurg. 2002;36(3):113-8.

6. Holmes AD, Meara JG, Kolker AR, Rosenfeld JV, Klug GL Fronthoetmoidal encephaloceles: reconstruction and refinements. J Craniofac Surg. 2001;12(1):6-18.

7. Tucker N, Chow D, Stockl F, Codere F, Burnier M. Clinically suspected primary acquired nasolacrimal duct obstruction: clinicopathologic review of 150 patients. Ophthalmology. 1997;104(11):1882-6. Review.

8. Fergie JE, Purcell K, Bishop J. Sepsis after nasolacrimal duct probing. Pediatr Infect Dis J. 2000;19(10):1022-3 